\title{
Action Trajectory Reconstruction for Controlling of Vehicle Using Sensors
}

\author{
Vani Prasanna . V ${ }^{1,}$ P.Srinivasulu ${ }^{2}$ \\ ${ }^{1}$ Department of Electronics and Communication Engineering \\ ${ }^{2}$ Sri Kalahasteeswara Institute of Technology, Sri kalahasti-517640, India
}

\begin{abstract}
Inertial sensors, such as accelerometers and gyro-scopes, are rarely used by themselves to compute velocity and position as each requires the integration of very noisy data. The variance and bias in the resulting position and velocity estimates grow un-bounded in time. This paper proposes a solution to provide a de-biased and de-noised estimation of position and velocity of moving vehicle actions from accelerometer measurements. The method uses a continuous wavelet transform applied to the measurements recursively to provide reliable action trajectory reconstruction. The results are presented from experiments performed with a MEMS accelerometer and gyroscope.
\end{abstract}

Keywords: Action trajectory, continuous wavelet transform, inertial measurement unit.

\section{Introduction}

Inertial sensors are primarily used for navigational purposes, but normally only as one component of a more complex system. Inertial sensors are typically complemented by GPS, video cameras, infrared, and other external speed, position and orientation sensors. Measurements are subject to very high noise, time varying bias and no internal mechanism to correct bias in position or velocity estimates. Recent advances in Micro electro mechanical systems (MEMS) technology have lead to low cost, small size, lightweight inertial measurement units (IMU). These advances are now being implemented in sports technology, video games, animation applications, robotic toys and mini-aerial vehicles. Inertial sensors are often used for orientation and balancing.

The proposed project considers simulating the control of Unmanned Vehicle maneuvering by means of detecting forearm movements using MEMS accelerometers. A three axis accelerometer is attached to the forearm and the forearm movements for different controls of the vehicle such as right turn, Left turn, forward movement and backward movement are detected by the accelerometer in 3-axis and the data is digitized and processed in the computer using MATLAB Soft-ware. Measurements are subject to very high noise, time varying bias and no internal mechanism to correct bias in position or velocity estimates. A de-biased and denoised estimation of position and velocity of forearm movements from accelerometer measurements are achieved. The signal processing method uses a continuous wavelet transform applied to the measurements recursively to provide reliable action trajectory reconstruction.

Raw measurements from the IMUs are supplied in a local and changing frame of reference, where the transformations between local and global reference frames are unknown, and have to be estimated from repeated measurement. Several tracking algorithms have been proposed. These estimate orientation. Complementary tracking on orthogonal groups [5] has been realized to a satisfactory performance, and is used.

In this paper we extend this approach, concentrating on computing the trajectories and velocities given acceleration measurements. A current approach, referred to as dead (sometimes also ded-for deduced) reckoning would compute velocity and position by integration and double integration of acceleration measurements. Unfortunately, the errors between actual and estimated position and velocity can become unbounded with time [9]. Effectively, the variance of the velocity grows with time as

$$
\sigma_{v}^{2}=\sigma^{2} \Delta t^{2} N
$$

and the variance of the position as

$$
\sigma_{v}^{2}=\sigma^{2} \Delta t^{4} N(N+1)(2 N+1),
$$

Where $\sigma^{2}$ is the acceleration variance, $\Delta t$ is the length of time step and $N$ is the number of time steps. Acceleration bias is time varying, and is commonly modeled by a slow growing exponential function. The integration of such bias causes large errors over time. It has been reported that the average displacement error for Xsens IMU's after 1 minute is 152 meters [10].

To overcome the bias/noise problems, attempts were made (such as in [11], [12]) not to perform integration at all, but to use acceleration measurements for the purpose of classification of actions. But this requires coping with the problem that different rates of movement along the same trajectory result in manifestly different acceleration measurements. This problem is overcome by the use of arc length (instead of time) to provide normalization, as arc length allows tracing of action curves with unit speed and permits partial action 
recognition. Unfortunately, computation of the arc length requires integration of the data and is therefore subject to the issues of noise and bias as described above.

Satisfactory trajectories were obtained when IMU's were complemented by GPS, infrared sensors, or other position giving sensors [13,14,15]. For our application if GPS is used their large current consumption will drain the battery's power quickly, limiting the length of acquisition. Use of a larger battery would be counter to the criteria of small size and unobtrusiveness. Optical sensors (as in the Wii remote) have an extremely limited volume of space in which the patient can be monitored, and require the patient to remain constantly within that limited space.

In [16] the periodic motion trajectory was computed using the parameterized model of periodic motion and the parameters were estimated from acceleration data.

In our approach, the approximate acceleration is obtained in a basis of continuous wavelets. After that, velocity and position are obtained through analytical integration.

In Section 2 we describe the problem and define action trajectories. In Section 3 we provide a brief description of continuous wavelet transforms, and the justification for a particular choice of basis that we use.

Section 4 gives a brief description of the complementary tracking filter. Section 5 describes the estimation algorithm for trajectory reconstruction. The data was collected using a low cost inertial measurement unit attached to a patient's wrist. The data was then processed using the algorithm described here. We present the results in Section 6.

\section{Statement of the Problem}

The accelerometer measurements can be represented mathematically by the following equation $a_{L}=R^{T}\left(a_{G}+g\right)+b_{a}+v_{-a}$,

Where $\mathrm{R}$ is a rotation matrix, representing transformation between local and global frames of reference, $\mathrm{a}_{\mathrm{G}}$ is the acceleration vector in the global frame, $\mathrm{g}$ is the gravity vector regarded as constant in this context, $b_{\mathrm{a}}$ is an acceleration measurement bias and $\underline{v}_{a}$ is the zero-mean wide-band noise with variance $\sigma^{2}$. The rotation matrix is time dependent and has to be estimated using all measurements from the inertial sensor. We use the complementary filter from [5] for tracking on the rotation group S0(3). It is defined as

$$
S O(3)=\left\{R \in R^{3} \mid R^{T}=R^{-1}\right\}
$$

The filter provides an estimation of the rotation matrix and has been realized, and gives a satisfactory performance. Given $\mathbf{R}$, an estimate of the acceleration $\mathrm{a}_{\mathrm{G}}$ is computed in a global frame, by the rotation of the acceleration measurements

And the subtraction of the gravity vector. An action is defined to be a smooth space curve: $\gamma$ :

$$
\left[t_{1}, t_{2}\right] \rightarrow R^{3} \text { such that }
$$

$\gamma\left(t_{1}\right)=\gamma:\left(t_{2}\right)=0$

$\gamma\left(t_{1}\right)=\gamma:\left(t_{2}\right)=0$

$$
\gamma(t) \neq 0 \quad t \in\left[t_{1}, t_{2}\right]
$$

That is, at the start time $t_{1}$ and end time $t_{2}$ the arm is completely static, and between those times the arm is in motion. Between $t_{1}$ and $t_{2}$ an action curve lies on a manifold and is definable in terms of the constraints imposed by the arm kinematics.

\section{Theory of Continuous Wavelet Transform}

In this section we describe the continuous wavelet transform (CWT). A CWT provides a redundant, but finely detailed, representation of a signal in terms of both time and frequency. CWTs are often used in problems involving signal identification and the detection of short duration elements of a signal, often hidden in noise.

The CWT is defined by a mother wavelet $\Psi(\mathrm{t})$, which has to satisfy a few properties, such as being absolutely and square integrable, having integral zero and a unit norm. In order to be invertible as a transform it also has to satisfy the admissibility condition [17]. It is often useful to restrict $\Psi$ (t) to be a continuous function with a number $\mathrm{M}$ of vanishing moments such that for all integers $\mathrm{m}<\mathrm{M}$

$\int_{R} t^{m} \Psi(t)=0$ function $\mathrm{f}(\mathrm{t})$.

This condition in particular allows the "filtering out" of all polynomials of degree $<M$ from the For a discrete signal $\mathrm{f}(\mathrm{t})$, the CWT is computed by correlation of the signal with functions $\Psi_{s}(\mathrm{t})$ defined by 


$$
\Psi_{s}(t)=\frac{1}{\sqrt{s}} \Psi\left(\frac{t}{s}\right),
$$

where $\mathrm{s}>0$ is the scale parameter which deter-mines the width of the wavelet. That is

$\Psi(s, b)=\int_{R} f(t) \frac{1}{\sqrt{s}} \Psi *\left(\frac{t-b}{s}\right) d t$

Where $*$ denotes complex conjugation. These correlations are computed for various scales and assembled as rows of a matrix which is referred to as scaleograms. The scaleogram essentially provides a measure of similarity between the signal $f(t)$ and the wavelet $\Psi(t)$ and its scaled shifted versions.

\section{Complementary Tracker}

Several algorithms are used to estimate the orientation of an IMU. The dead reckoning method incorporates changes given by the gyroscope to an attitude at the previous epoch. Dead reckoning suffers the most from the accumulation of errors [10]. Commonly found in current systems is another approach using extended stochastic linear estimation techniques $[6,7,8]$. However, the Gaussian noise assumptions of such filters do not apply well, and frequent re initialization is required. The complementary tracker [5] is designed for tracking on the rotation group, defined in Eq. (4). It finds a mapping from the local frame of the IMU to the global frame of reference in terms of a rotation Matrix $\mathrm{R}$ or equivalently a quaternion. The quaternion representation of rotations is commonly used for the realization of algorithms on $\mathrm{SO}(3)$ because of its continuity and efficiency in implementation. The set of quaternion's is denoted

$$
\mathrm{Q}=\left\{\mathrm{q}=(v ; \mathrm{s}) \in \mathrm{R}^{3} \mathrm{xR}:|\mathrm{q}|=1\right\}
$$

where $\mathrm{v}=\left[\begin{array}{lll}\mathrm{v}_{1} & \mathrm{v}_{2} & \mathrm{v}_{3}\end{array}\right]^{\mathrm{T}}$ and $\mathrm{s}$ are respectively the vectors and the scalar part of the quaternion. The identity element of the quaternion's is $\left[\begin{array}{llll}0 & 0 & 0 & 1\end{array}\right]^{\mathrm{T}}$. In the methodology the following assumptions are used

$$
\begin{aligned}
& \frac{\mathrm{a}}{|\mathrm{a}|} \approx \mathrm{R}^{\mathrm{T}} \mathrm{e}_{\mathrm{z}} \\
& \mathrm{m} \approx \mathrm{R}^{\mathrm{T}} \mathrm{h} \\
& \frac{\mathrm{dR}}{\mathrm{dt}}=\Omega \mathrm{xR}
\end{aligned}
$$

where a and $\mathrm{m}$ are respectively accelerometer and magnetometer measurements, $\mathrm{e}_{\mathrm{z}}$ is a unit vector in the direction of the gravity vector $\mathrm{g}$, i.e. downwards, $\mathrm{h}$ is the direction of the magnetic field unit vector and $\Omega$ is the rotation rate vector given by gyroscope measurements. It is assumed that the gravity and magnetic field vectors are both constant over time. At each epoch the quaternion is initially computed using the accelerometer and magnetometer measurements via maximum likelihood estimation techniques [18], i.e.

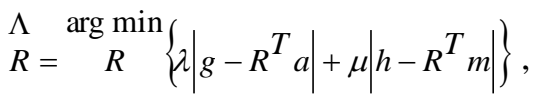

where the weights $\lambda$ and $\mu$ represent the confidence in a particular measurement.

Innovation $\omega$ on the rotation group is then computed as follows

$$
\begin{aligned}
& \tilde{\mathrm{R}}={\stackrel{\mathrm{R}^{\mathrm{R}}}{\mathrm{T}} \mathrm{R}}^{\mathrm{R}}=\operatorname{vex}\left(\frac{1}{2}\left(\tilde{\mathrm{R}}-\tilde{\mathrm{R}}^{\mathrm{T}}\right)\right),
\end{aligned}
$$
matrix $\mathrm{W}$.

where vex (W) denotes the inverse of the vector cross product operator, realized by a skew-symmetric

The final estimates for the rotation matrix and gyroscope bias are obtained using the filter innovation and gyroscope measurements as follows. 


$$
\begin{aligned}
& \frac{d b}{d t}=-2 k_{1} \omega \\
& \Lambda=\Omega-b+2 k_{2} \omega \\
& \frac{d R}{d t}=\stackrel{\Lambda}{\Omega} \times R
\end{aligned}
$$

where $\mathbf{b}$ is the filter estimate of the gyroscope bias, $\stackrel{\Lambda}{\Omega}$ is the filter estimate of the actual angular velocity and $\mathrm{k}_{1}$ and $\mathrm{k}_{2}$ are experimentally chosen constant filter gains.

We have realized this filter to a satisfactory performance, however the filter does not provide any measure of uncertainty of the estimated rotation, nor does it compute the filter gain. Some work is currently underway to resolve these problems and so further improve the algorithm.

\section{Residual Cwt Algorithm Description}

Acceleration is provided as a time varying vector, where vector elements are respectively the $\mathrm{x}, \mathrm{y}, \mathrm{z}$ coordinates of the vector. We apply identical processing to each vector element, and therefore it is sufficient to regard the acceleration as a time series. Our aim is to approximate each of the components in terms of a continuous wavelet basis;

that is, of the form

$a(t)=\sum_{i} \beta_{i} \frac{1}{\sqrt{s_{i}}} \psi\left(\frac{t-b_{i}}{s_{i}}\right)$

Where $\beta_{i}^{\prime} \mathrm{s}, s_{i}^{\prime} \mathrm{s}$, and $b_{i}^{\prime} \mathrm{s}$ are respectively coefficients, scale and shift parameters of wavelet decomposition. It seems logical that the choice of basis should depend on the application. In our case we choose the basis function $\Psi$ (mother wavelet) to be the first order Hermitian wavelet, defined as

$$
\psi(t)=-2\left(\frac{2}{\pi}\right)^{\frac{1}{4}} t \exp \left(-t^{2}\right)
$$

this is a normalized derivative of the Gaussian function. Inspection of the data suggests that this is a good choice of basis to represent the acceleration, although other choices are possible. The wavelet is essentially compactly supported having Gaussian decay, and hence property Eq. (5) of action curve can be effectively satisfied. The integral of this wavelet is a Gaussian function which is also essentially compactly supported, hence the second and the third properties Eq. (6) and Eq. (7) are also satisfied. The wavelet has zero vanishing moments, which means it can eliminate any constant bias from the acceleration data without the need to estimate it. We seek the set of triples $\left\{\beta_{\mathrm{i}}, \mathrm{s}_{\mathrm{i}}, \mathrm{b}_{\mathrm{i}}\right\}_{i=1 \ldots \ldots N}$, such that the Eq. 16 gives us the desired accuracy to represent acceleration data. Note that $\mathrm{N}$ is unknown and needs to be estimated from the data too. Simple thresholding of the scaleogram of the acceleration data can lead to unsatisfactory performance, as it is not clear how to choose a suitable threshold since the additive noise variance is unknown. In out approach, we use a recursive procedure in which the values of interest are determined from the scaleogram and the corresponding components are then removed from the data. The recursion is terminated when the maximum peak of the scaleogram has fallen below a threshold. The threshold is chosen so that the position is reconstructed with the desired level of detail and accuracy. Another way to terminate the recursion is to test the residual acceleration for normality. Once the residual acceleration is detected to have a normal distribution it is assumed to be at the noise level and recursion is terminated. Both methods lead to a satisfactory and similar performance. Velocity and position are then computed analytically using the set of $\mathrm{N}$ triples for a sampling frequency $\mathrm{F}$ as follows

$$
\begin{aligned}
& v(t)=\frac{1}{F} \sum_{i=1}^{N} \beta_{i} s_{i}^{\frac{1}{2}}\left(\frac{2}{\pi}\right)^{\frac{1}{4}} \exp -\left(\frac{\left(t-b_{i}\right) 2}{s_{i}^{2}}\right) \\
& \gamma(t)=\frac{1}{2 F^{2}} \sum_{i=1}^{N} \beta_{i} s_{i}^{\frac{3}{2}}(2 \pi)^{\frac{1}{4}} \operatorname{erf}\left(\frac{\left.t-b_{i}\right)}{s_{i}}\right)
\end{aligned}
$$

The procedure is summarized by Algorithm 5.1.

Algorithm 5.1: RESIDUAL CWT $(\psi)$

comment: Compute the set of triple parameters from acceleration

$V \leftarrow \theta$ 
While true

do $\left\{\begin{array}{l}\text { Compute scaleogram } \Phi(\mathrm{s}, \mathrm{b}) \\ \text { Select maximum value of the scaleogram } \\ \beta_{0}=\max (\Phi) \\ \text { it takes the maximum value at } \mathrm{s}_{0} \text { and } \mathrm{b}_{0}\end{array}\right.$

if $\beta_{0} s_{0} \frac{3}{2}>$ threshold

then $\left\{\begin{array}{l}v=v U\left\{\beta_{0}, s_{0}, b_{0}\right\} \\ \text { remove the component from the data } \\ a(t) \leftarrow a(t)-\beta_{0} \Psi_{s_{0}}\left(t-b_{0}\right)\end{array}\right.$

else break

return $(V)$

VI. Experimental Results

Experiments were conducted with a commercial inertial measurements unit X sens.

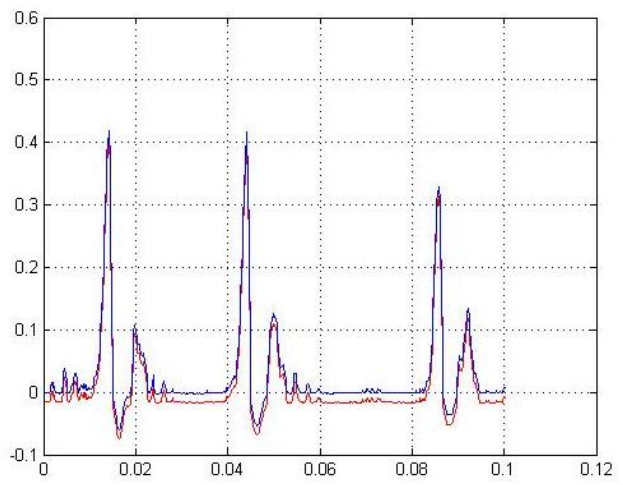

Fig. 1. Wavelet reconstructed signal for forward movement

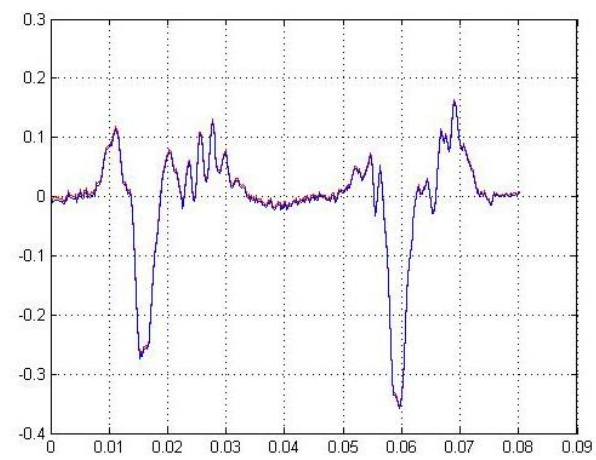

Fig. 2. Wavelet reconstructed signal for backward movement

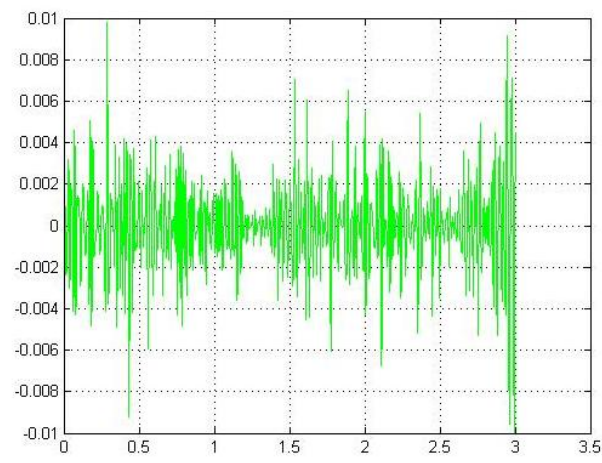

Fig. 3. Wavelet reconstructed signal for right movement 


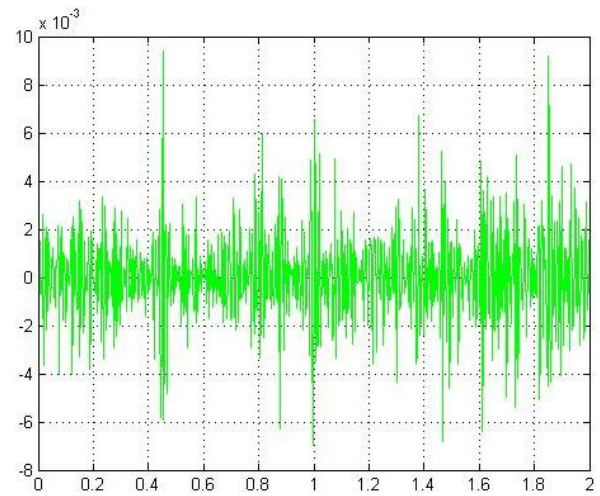

Fig. 4. Wavelet reconstructed signal for left movement

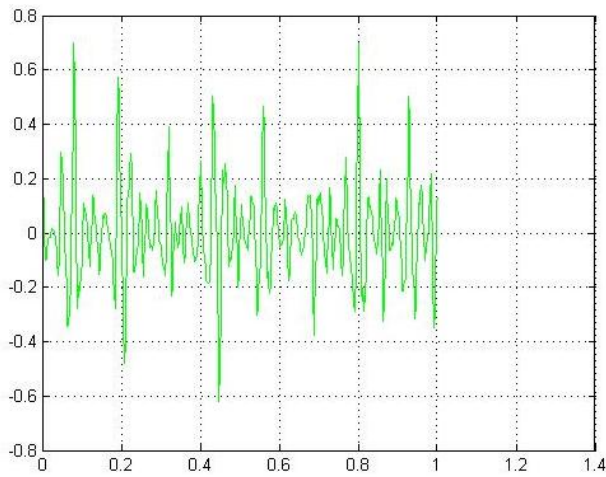

Fig. 5. Wavelet reconstructed signal to stop

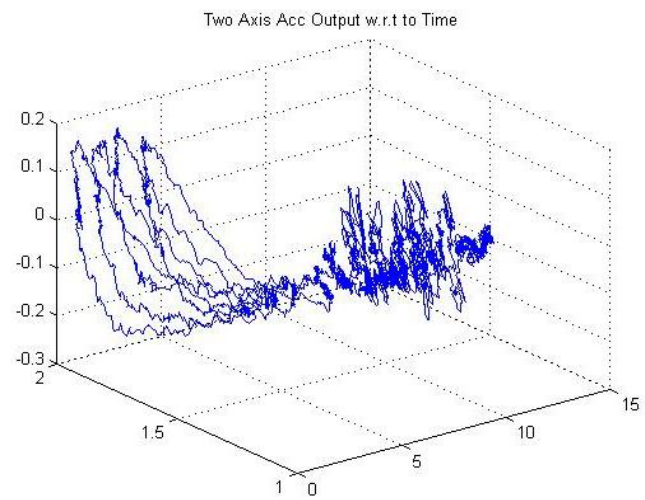

Fig. 6. 3D plot for 3-axis MEMS accelerometer

\section{Conclusion}

In this paper an algorithm to compute the trajectory of the action from acceleration measurements is proposed. The problem is posed as an estimation problem and uses continuous wavelet transforms applied to the data. The estimates are shown to be essentially bias-free, and provide sufficient grounds for subsequent action recognition and classification. Preliminary experiments were conducted to demonstrate the effectiveness of the algorithm. The experiments involved simple trajectories, and more experiments with more complex trajectories are underway. In order to compute errors and statistics, further experiments are conducted and the results will be reported.

\section{References}

[1] Kevin King, S.W. Yoon, N.C. Perkins, K. Najafi, "Wireless MEMS inertial sensor system for golf swing dynamics", Sensors and Actuators A: Physical, vol.141,2,2008.

[2] Tapus, Tapus, Mataric, "Userrobot personality matching and assistive robot behavior adaptation for poststroke rehabilitation therapy", Intelligent Service Robotics, vol. 1, issue.2, 2008.

[3] Kelly, Saripalli, Sukhatme, "Combined Visual and Inertial Navigation for an Unmanned Aerial Vehicle," Field and Service Robotics, vol.42,2008 @ 
[4] Cha, ByungRae. and Jung, YoungKee., "Gesture Recognition Based on Motion Inertial Sensors for Ubiquitous Interactive Game Contents", IETE Technical Review, vol. 27, n. 2, 2010

[5] Robert Mahony, Tarek Hamel, Jean-Michel Pflimlin, "Non-linear complementary filters on the special orthogonal group," IEEE Transactions on Automatic Control 53, 5, 2008

[6] Angelo M. Sabatini, "Quaternion-Based Extended Kalman Filter for Determining Orientation by Inertial and Magnetic Sensing”, ieee transactions on biomedical engineering, vol. 53, NO. 7, July 2006.

[7] Zhang, S., Ang, M.H., Xiao, W., Tham, C.K. "Detection of activities for daily life surveillance: Eating and drinking," e-health Networking, Applications and Services. HealthCom 2008. $10^{\text {th }}$ International Conference on, 2008

[8] Hassen Fourati, Noureddine Manamanni, Lissan Afilal, Yves Handrich, "A Nonlinear Filtering Approach for the Attitude and Dynamic Body Acceleration Estimation Based on Inertial and Magnetic Sensors: Bio-Logging Application,” ieee sensors journal, vol. 11, no. 1, Jan. 2011

[9] John H. Wall and David M. Bevly, "Characterization of Inertial Sensor Measurements for Navigation Performance Analysis," Proceedings of the 19th Interna-tional Technical Meeting of the Satellite Division of 993 The Institute of Navigation (ION GNSS 2006), Fort Worth, TX, Sep. 2006

[10] Oliver J. Woodman, "An introduction to inertial navigation," Technical Report Number 696, University of Cambridge, 2007, http://www.cl.cam.ac.uk/techreports/ 